\section{REPENSANDO LA EDUCACIÓN DE LOS PROFESIONALES DE SALUD DEL SIGLO XXI: CAMBIOS Y ACCIONES EN UN MUNDO GLOBAL}

\section{RETHINKING THE EDUCATION OF XXI CENTURY HEALTH PROFESSIONALS: CHANGES AND ACTIONS IN A GLOBAL WORLD}

\author{
Patricia J. García $a^{1, a, b}$
}

Sr. Editor. Recientemente se ha publicado en la prestigiosa revista médica Lancet, un artículo en inglés titulado Profesionales de salud para nuevo siglo: Transformando la educación para fortalecer los sistemas de salud en un mundo interdependiente ${ }^{(1)}$, el cual es un reporte de una comisión en la que tuve la oportunidad de participar, y el que, a propósito de cumplirse los 100 años del prestigioso Informe Flexner ${ }^{(2)}$, cambió la educación en las facultades de medicina; en él se analizan las necesidades en la educación de profesionales de salud en el siglo XXI. Ya era tiempo que nos planteáramos globalmente el reto de repensar qué estamos haciendo en el entrenamiento de nuestros profesionales de salud, qué hemos logrado, qué falta y qué necesitamos hacer; dicho reporte discute todos estos aspectos. También se incluye en este número de la revista, el resumen ejecutivo del panel que sintetiza las reformas propuestas. Sin embargo, quisiéramos destacar los principales mensajes y aportes de la comisión que, esperamos, puedan ser discutidos y finalmente implementados por los diversos actores relacionados con salud y educación de los profesionales del campo en nuestro país y en la región.

Con una visión que incluye a diversas ramas relacionadas con la salud, y no solo a la medicina, el reporte presenta la necesidad de cambios, dados los problemas de currículos fragmentados, estáticos, que no incluyen la lógica de sistemas de salud y con contenidos alejados de los nuevos retos de este siglo. Hoy en día se presentan grandes demandas para los profesionales de salud en un mundo global interdependiente: infecciones nuevas o reemergentes, riesgos ambientales, enfermedades crónicas, enfermedades mentales, violencia, accidentes y envejecimiento de las poblaciones. Por si fuera poco, los sistemas de salud no se han alineado aún a estos retos. En este panorama cambiante, es una

Facultad de Salud Pública y Administración. Universidad Peruana Cayetano Heredia. Lima, Perú.

a Master of Public Health; ${ }^{b}$ Doctor en Medicina.

Recibido: 30-03-11 Aprobado: 06-04-11 necesidad imperiosa preparar a los profesionales de la salud para afrontar el nuevo milenio y para ser ellos mismos agentes de cambio. Para ello, la comisión propone el uso de una "enseñanza transformativa".

El reporte reconoce la actual división de las profesiones en salud como una construcción social, asociada con el progreso científico, pero también a relaciones económicas, intereses políticos, cultura y luchas por delimitar su ámbito de acción. Esta división o "compartamentalización" profesional lleva a conflictos, aislamiento e ineficiencia y no es "saludable" para el trabajo en el campo de la salud. La Comisión propone la evolución hacia una educación interdisciplinaria, incluso transdisciplinaria, que facilite el trabajo en equipo clave en salud.

El artículo presenta un análisis basado en un marco de sistemas en el que se realza las complejas y necesarias relaciones entre los sistemas de educación y salud y su interdependencia, teniendo como motor fundamental a la población a la que sirven.

Se reconoce que las instituciones educativas son elementos cruciales para transformar los sistemas de salud, estas pueden generar evidencias respecto a problemas en los sistemas de salud y posibles soluciones y también pueden (y debieran) producir profesionales capaces de implementar cambios. Por otro lado, los sistemas de salud tienen necesidades y el sistema educativo debiera ser capaz de entender y responder a sus requerimientos, tanto en números como en destrezas requeridas por los profesionales para cumplir con sus funciones. Este es, desde donde se mire, un balance crítico y clave que requiere el trabajo de ambos sistemas: salud y educación, de manera más estrecha y coordinada.

El marco de análisis que se usa en el reporte, identifica tres dimensiones clave en la educación en salud: el diseño institucional (es decir, la estructura y funciones del sistema educativo); el diseño instructivo (es decir, los procesos); y los resultados deseados. Si bien los reportes de hace más de un siglo, Flexner en educación médica (2), Welch-Rose en salud pública ${ }^{(3)}$ y Goldmark (enfermería), incluyeron aspectos sobre qué y cómo enseñar (diseño instructivo), y también algunos temas sobre dónde enseñar, (es decir aspectos del diseño institucional), el actual informe aporta la visión de sistemas, de organizaciones interrelacionadas como parte de un sistema educativo. Se plantea una perspectiva global, con cuatro funciones fundamentales para el diseño institucional (1) responsabilidad administrativa ${ }^{(2)}$ financiamiento ${ }^{(3)}$ generación de recursos y provisión de servicios ${ }^{(4)}$; y se discuten nuevas estrategias dirigidas a mejorar los procesos instructivos, desde los criterios de admisión, pasando por las competencias y líneas de carrera. 
El reporte se enriquece de un diagnóstico global basado en las categorías del marco conceptual, analiza instituciones, graduandos, financiamiento en diversos países y en todas las regiones del mundo. También presenta información sobre diseños instructivos y ejemplos del uso de las tecnologías de información y comunicación (TIC) para la educación abierta, la globalización de la salud y la enseñanza de la salud global. Se enfatiza la importancia de buscar la promoción del aprendizaje transformacional, es decir aquel que promueve el desarrollo de atributos de liderazgo y la formación de agentes de cambio y el reconocimiento de la interdependencia en educación, para la equidad en salud, centrada en el paciente y basada en la población.

Finalmente, la comisión no pretende haber resuelto las preguntas, sino, pone sobre la mesa de discusión las necesidades de cambio en la educación de los profesionales de salud que confrontamos en este nuevo siglo en un mundo global e interdependiente. El documento termina invitando a la acción a los actores relacionados con la educación de profesionales en salud, a los estudiantes y jóvenes profesionales, a las organizaciones profesionales, gobiernos, ONG, agencias internacionales, donantes y fundaciones. Se hace un llamado a la discusión, al reconocimiento de los retos y a la búsqueda de soluciones, al intercambio y al debate sobre estas recomendaciones y, finalmente, a realizar acciones concretas que permitan que la interacción entre los sis- temas de salud y educación encuentren el dialogo y el balance que requieren para lograr su cometido de un progreso equitativo en salud.

\section{Conflictos de Interés}

La autora declara no tener conflictos de interés.

\section{REFERENCIAS BIBLIOGRÁFICAS}

1. Frenk J, Chen L, Bhutta ZA, Cohen J, Crisp N, Evans T, et al . Health professionals for a new century: transforming education to strengthen health systems in an interdependent world. Lancet. 2010 Dec 4;376(9756):1923-58.

2. Flexner A. Medical education in the United States and Canada: a report to the Carnegie Foundation for the Advancement of Teaching. New York: Carnegie Foundation for the Advancement of Teaching; 1910.

3. Welch WH, Rose W. Institute of Hygiene: a report to the General Education Board of Rockefeller Foundation. New York: The Rockefeller Foundation; 1915.

4. The Committee for the Study of Nursing Education. Nursing and nursing education in the United States. New York: The Rockefeller Foundation; 1923.

Correspondencia: Patricia J. García.

Dirección: Facultad de Salud Pública y Administración

Universidad Peruana Cayetano Heredia. Av. Honorio Delgado 430, Lima 31, Perú.

Teléfono: (511) 319-0028

Correo electrónico: patricia.garcia@upch.pe

\section{Consulte la versión electrónica de la Revista Peruana de Medicina Experimental y Salud Pública en}

wWW.scopus.com

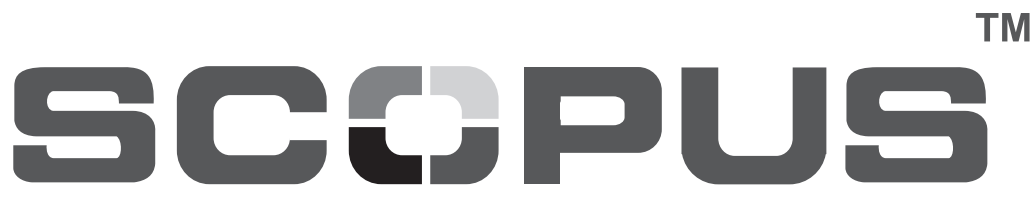

